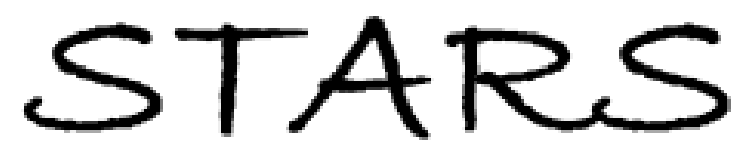

University of Central Florida

STARS

$1-1-1999$

\title{
Visible light emission from dyes excited by simultaneous absorption of two different frequency beams of light
}

\author{
Alexandra Rapaport \\ University of Central Florida \\ Karine Ayrault \\ University of Central Florida \\ Eyitope St. Matthew-Daniel \\ University of Central Florida \\ Michael Bass \\ University of Central Florida
}

Find similar works at: https://stars.library.ucf.edu/facultybib1990

University of Central Florida Libraries http://library.ucf.edu

This Article is brought to you for free and open access by the Faculty Bibliography at STARS. It has been accepted for inclusion in Faculty Bibliography 1990s by an authorized administrator of STARS. For more information, please contactSTARS@ucf.edu.

\section{Recommended Citation}

Rapaport, Alexandra; Ayrault, Karine; Matthew-Daniel, Eyitope St.; and Bass, Michael, "Visible light emission from dyes excited by simultaneous absorption of two different frequency beams of light" (1999). Faculty Bibliography 1990s. 2801.

https://stars.library.ucf.edu/facultybib1990/2801

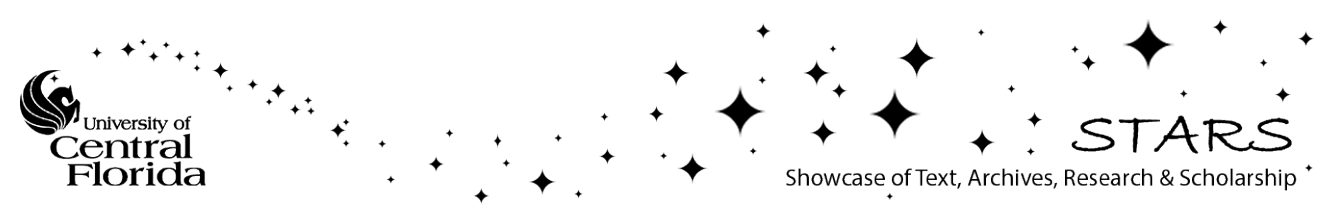




\section{Visible light emission from dyes excited by simultaneous absorption of two different frequency beams of light}

Cite as: Appl. Phys. Lett. 74, 329 (1999); https://doi.org/10.1063/1.123061

Submitted: 17 September 1998 . Accepted: 12 November 1998 . Published Online: 13 January 1999

Alexandra Rapaport, Karine Ayrault, Eyitope St. Matthew-Daniel, and Michael Bass
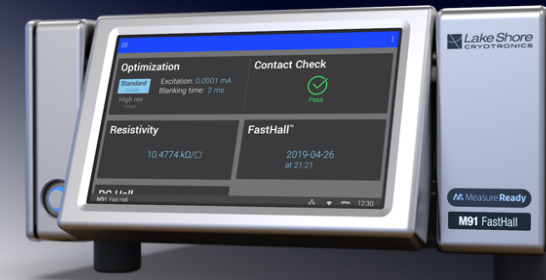

\section{Measure Ready} M91 FastHall ${ }^{\text {TM }}$ Controller

A revolutionary new instrument for complete Hall analysis

See the video 0

Lake Shore 


\title{
Visible light emission from dyes excited by simultaneous absorption of two different frequency beams of light
}

\author{
Alexandra Rapaport, Karine Ayrault, Eyitope St. Matthew-Daniel, and Michael Bass ${ }^{a}$ \\ School of Optics and Center for Research and Education in Optics and Lasers (CREOL), \\ University of Central Florida, Orlando, Florida 32816-2700
}

(Received 17 September 1998; accepted for publication 12 November 1998)

Emission of visible light by dyes in both liquid and polymer hosts is reported when excited by simultaneous absorption of infrared light from two different wavelength sources. By moving the intersection point of these two beams about within the volume of the dye-doped medium, one can create a true, real-time, three-dimensional display. (c) 1999 American Institute of Physics.

[S0003-6951(99)02903-4]

Excitation of visible light emission by dyes in both liquid and polymer hosts is demonstrated when the dye is excited by simultaneous absorption of light at two different infrared frequencies. While the materials studied were not optimized, the results achieved prove the feasibility of using dye-doped media in both two- and three-dimensional (2D and 3D) displays. Steps necessary to optimize dye-doped materials for these applications are identified.

The infrared frequencies for nonlinear excitation were chosen so that neither one can experience two-photon absorption alone. This was achieved by selecting the frequencies of the pump sources so that the second harmonic of one was too high to be absorbed by the dye and the second harmonic of the other was too low. However, the sum frequency of the two infrared sources corresponds to a light frequency that lies within the dye's absorption band. It is the simultaneous absorption of two different frequency photons from the two different sources that gives rise to visible emission only in the region where the two beams overlap. This is a form of two-photon absorption-excited emission in the dye solution (two-photon absorption in molecular systems has been studied since the early 1970 's ${ }^{1,2}$ ), and has the potential to make possible real-time, multicolor three-dimensional displays. While doped crystals and glasses have been suggested for and demonstrated in three-dimensional displays, ${ }^{3,4}$ fabricating large volumes of these materials presents great technological difficulties, and the product achieved would be of prohibitive weight and cost. On the other hand, liquid dye solutions do not suffer these restrictions. More convenient and similarly scalable are dye-doped polymers. In this letter we present data on two-photon absorption-excited visible emission from dye-doped polymers and show how they can be used to make multicolor three-dimensional displays. It should be noted that dye-doped polymers using this process for excitation can be readily used in large-area twodimensional displays.

Figure 1 is useful in understanding the model for twophoton absorption using two different frequency (wavelength) light sources in an idealized dye solution. Twophoton absorption will take place if the energy of the two photons corresponds to an energy absorbed by the dye. Thus, as indicated in Fig. 1, the infrared sources are chosen so that

${ }^{a)}$ Electronic mail: bass@creol.ucf.edu twice the energy of neither source lies in the dye solution's absorption band but that the sum of the energy of one photon from each source does. Since most dye solutions efficiently fluoresce upon absorbing light, wherever the appropriate two different frequency photons are simultaneously present there will be light emission from the excited volume.

Linear absorption spectra were measured using a dualbeam spectrophotometer (Perkin-Elmer 330) for both liquid solutions and dye-doped polymers. Excitation spectra were measured using a two double-grating monochromator configuration. Emission spectra were recorded using one doublegrating monochromator. The results of such measurements for a solution of $1 \times 10^{-5} \mathrm{M}$ pyrromethene in methanol are given in Fig. 2.

Two-photon absorption experiments were performed using either a $Q$-switched neodymium-doped yttrium aluminum garnet $\left(\mathrm{Nd}^{3+}\right.$ :YAG) laser, or a $Q$-switched chromiumdoped lithium strontium aluminum fluoride $\left(\mathrm{Cr}^{3+}: \mathrm{LiSAF}\right)$ laser, or both depending on the peak absorption wavelength of the dye studied. Visible emission was detected through a monochromator by a type I photomultiplier. The pump pulses were monitored by fast $P I N$ photodiodes having 0.5 ns response times.

In order to demonstrate the absorption of two photons of the same wavelength in the dye solutions and in the dyedoped polymers, we needed to prove the dependence of the

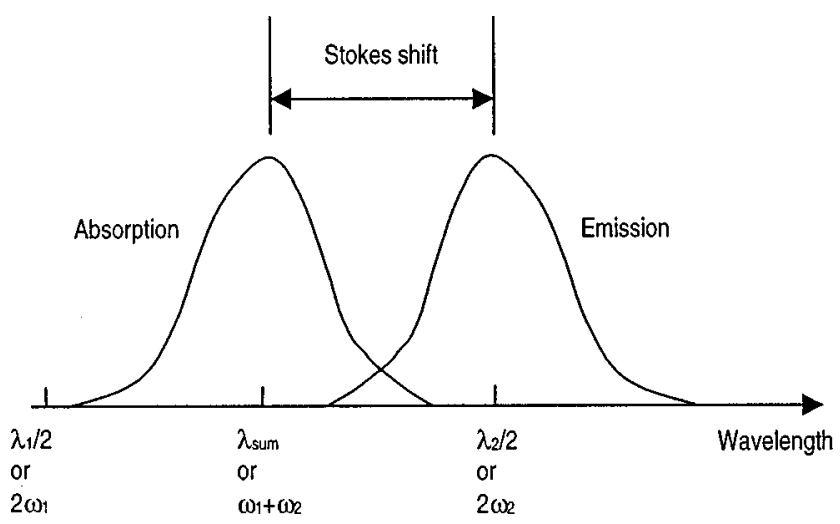

FIG. 1. A schematic plot of the absorption and emission spectra of an idealized dye solution vs wavelength. Shown in this sketch are the desired locations of the second harmonics of the pump sources and their sum frequency. The Stokes Shift between absorption and emission peaks is indicated. 


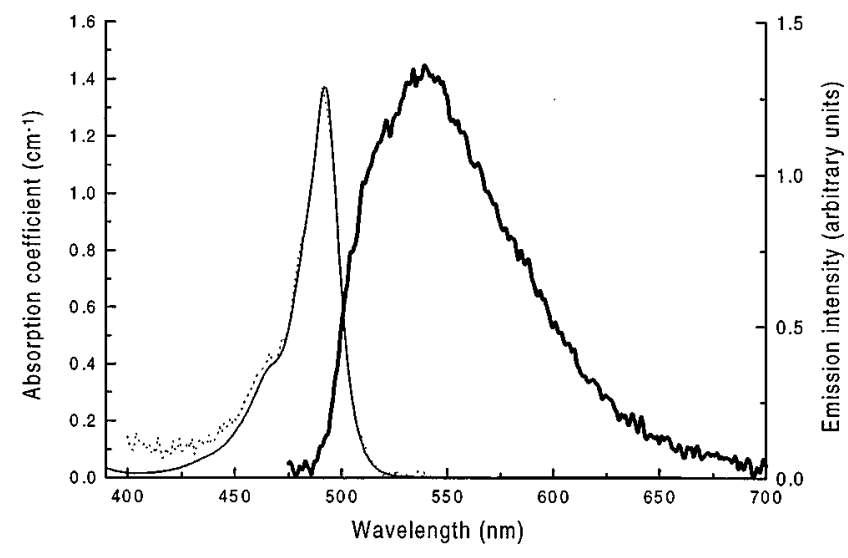

FIG. 2. Absorption, excitation, and emission spectrum of a solution of 1 $\times 10^{-5} \mathrm{M}$ of pyrromethene in methanol. Solid line, coefficient of linear absorption in $\mathrm{cm}^{-1}$; dashed line, excitation (detection at $575 \mathrm{~nm}$ ); and bold solid line, emission (excitation at $450 \mathrm{~nm}$ ).

visible emission intensity on the square of the intensity of the pump. The necessary proof in both liquid solutions and dyedoped polymers was obtained by varying the peak pump intensity and monitoring the resulting peak intensity of the signal. Calibrated neutral density filters were placed in the pump beam in these experiments. Data obtained from such measurements for a solution of $5 \times 10^{-4} \mathrm{M}$ rhodamine pumped by the $Q$-switched $\mathrm{Nd}^{3+}$ :YAG laser are shown in Fig. 3(a) and demonstrated the square-law relationship. Additional confirmation was obtained since our detection system was fast enough to show that the signal wave form (e.g., the visible light intensity as a function of time) follows exactly the square of the pump pulse wave form. This type of data for the same dye solution is given in Fig. 3(b). It is clear that the visible emission observed is due to two-photon absorption of the single pump source.

Two-photon absorption of a single infrared pump wavelength was observed in several of the dye-doped polymers (see Table I). Some of the samples showed very strong visible emission and some much weaker. These plastics were designed for use in advertising displays and were not optimized for the present study. As a result, quantitative measurement of the emitted light was deferred until improved materials are received. In Table I, the blue CYRO plastics 6122-8 and 6141-8 are seen to have produced no visible emission under excitation at $850 \mathrm{~nm}$. However, these two samples emitted quite well in the blue when excited with the $\mathrm{Cr}^{3+}$ :LiSAF laser tuned to $800 \mathrm{~nm}$ (their absorption coefficient at $400 \mathrm{~nm}$ is about $20 \mathrm{~cm}^{-1}$ ). As a result, visible emission by dye-doped polymers of blue, green, yellow, and red light was obtained.

Experiments to show the absorption of two photons of two different infrared wavelengths in the dye solutions were conducted using both the Nd:YAG and Cr:LiSAF lasers. The use of a Stanford Research System model DG535 pulse generator to $Q$ switch both lasers resulted in very little interpulse jitter.

Figure 4 demonstrates the visible emission by simultaneous absorption of two different frequency infrared light sources. In it, traces show no visible emission for the 1064 $\mathrm{nm}$ pump source alone and very little when using $805 \mathrm{~nm}$
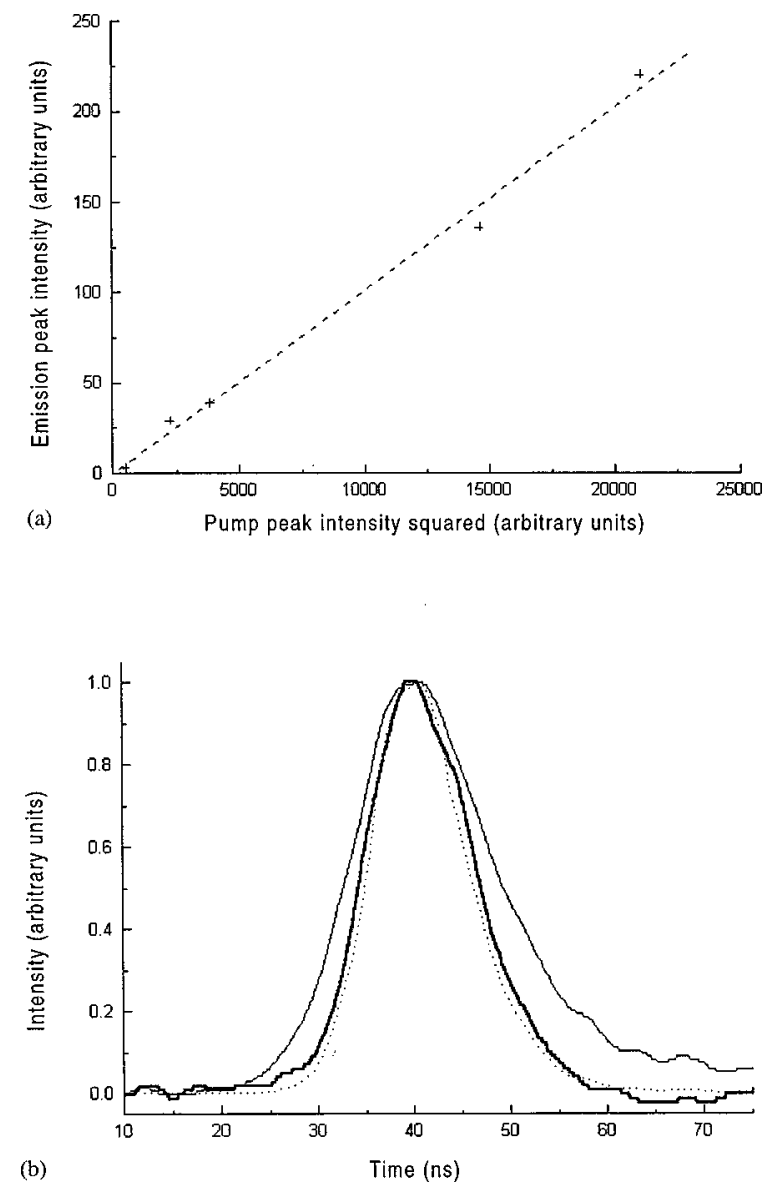

FIG. 3. (a) Visible emission peak intensity vs the square of the pump peak intensity in a $5 \times 10^{-4} \mathrm{M}$ solution of rhodamine in ethanol. The pump was a $\mathrm{Nd}^{3+}$ :YAG laser and the light emitted by the rhodamine was centered at $578 \mathrm{~nm}$. The + signs indicate measured values and the dashed straight line shows the square-law relationship. (b) Two-photon absorption excitation in a $5 \times 10^{-4} \mathrm{M}$ solution of rhodamine in ethanol. Bold solid line, visible emission from rhodamine detected at $578 \mathrm{~nm}$; solid line, $\mathrm{Nd}^{3+}:$ YAG pulse wave form at $1.064 \mu \mathrm{m}$; and dashed line, square of the $\mathrm{Nd}^{3+}$ :YAG pulse.

alone. However, when both are delivered simultaneously there is strong visible light emission.

It should be noted that in some dye solutions with too small a Stokes shift, overlap between the absorption and emission regions causes the color of the observed visible emission to depend on the location of the emitting site within the sample. Thus, a solution that emits yellow light near the surface appears to emit a more reddish color when excited in the center of the sample. To reduce this effect, dye solutions with large Stokes shift (such as obtained in solutions of coumarin dyes) will be more attractive candidates for 3D display applications.

The experiments reported show that dyes in liquid and polymeric solutions can be excited to emit visible light by simultaneous absorption of two different infrared light sources. Since the region of overlap of two such beams can be moved around within the volume of such a medium, the visible emission can be made to trace out a threedimensional image. The result would be a real-time, threedimensional display.

Some of the properties that an appropriately chosen dye should exhibit have already been identified. It should present a large Stokes shift and a narrow absorption band. It should also have a high coefficient of nonlinear absorption as well 
TABLE I. List of dye-doped polymers tested and their properties. The visually observed emission strength is indicated by no asterisks for no emission to three for strong emission. The nonlinear emission was observed either with a $1064 \mathrm{~nm} \mathrm{Nd:YAG} \mathrm{laser} \mathrm{focused} \mathrm{to} \mathrm{about} 100 \mathrm{MW} / \mathrm{cm}^{2}$ within the sample or a $850 \mathrm{~nm} \mathrm{Cr:LiSAF}$ laser focused to $30 \mathrm{MW} / \mathrm{cm}^{2}$.

\begin{tabular}{|c|c|c|c|c|c|}
\hline $\begin{array}{c}\text { CYRO }^{\mathrm{a}} \\
\text { LISA plastics } \\
\text { sheet number } \\
\text { and color }\end{array}$ & $\begin{array}{l}\text { Emission } \\
\text { color }\end{array}$ & $\begin{array}{c}\text { Absorption } \\
\text { coefficient at } \\
532 \mathrm{~nm}\left(\text { in }^{-1}\right)\end{array}$ & $\begin{array}{c}\text { Visible emission } \\
\text { under Nd:YAG } \\
\text { excitation }\end{array}$ & $\begin{array}{c}\text { Absorption } \\
\text { coefficient near } \\
425 \mathrm{~nm}\left(\text { in } \mathrm{cm}^{-1}\right)\end{array}$ & $\begin{array}{l}\text { Visible emission } \\
\text { under Cr:LiSAF } \\
\text { excitation }\end{array}$ \\
\hline $\begin{array}{c}6122-8 \\
\text { blue }\end{array}$ & Blue & $<0.5$ & & 0.6 & \\
\hline $\begin{array}{c}6141-8 \\
\text { blue }\end{array}$ & Blue & $<0.5$ & & 0.6 & \\
\hline $\begin{array}{l}\text { GPF 564-9 } \\
\text { green }\end{array}$ & Green & $<0.5$ & & 7 & $* *$ \\
\hline $3105-5$ & Yellow & 22 & $*$ & 2 & * \\
\hline $\begin{array}{c}\text { yellow orange } \\
411-5 \\
\text { orange pink }\end{array}$ & Yellow & 26 & $* *$ & 1 & $* * *$ \\
\hline $\begin{array}{c}T \text {-square } \\
\text { orange }\end{array}$ & Yellow & $>40$ & $* * *$ & 2 & $* * *$ \\
\hline $\begin{array}{c}2124-3 \\
\text { dark orange }\end{array}$ & Orange & $>28$ & $* * *$ & 13 & * \\
\hline $\begin{array}{c}2123-2 \\
\text { orange red }\end{array}$ & Orange & $>32$ & $* * *$ & 12 & $* *$ \\
\hline $\begin{array}{c}216-4 \\
\text { pink }\end{array}$ & Orange & 20 & $*$ & 2 & $* * *$ \\
\hline $\begin{array}{c}2130-2 \\
\text { pink red }\end{array}$ & Yellow red & 8 & & 3 & $* *$ \\
\hline $\begin{array}{c}2135-1 \\
\text { red }\end{array}$ & Yellow red & 16 & $*$ & 7 & $* *$ \\
\hline
\end{tabular}

${ }^{\mathrm{a} C Y R O}$ Industries, 25 Executive Blvd., P.O. Box 550, Orange, CT 06477-0550.

as a high quantum efficiency. However, more research remains to be done to optimize the dye and the host material. The samples studied showed that laser damage was an issue with the laser pulse energies used in the demonstration. Attention should be given to make polymers free of scattering. The use of mode-locked diode lasers should enable the intensities necessary for effective nonlinear excitation of dyedoped media.

Liquid display media could be difficult to handle and possibly dangerous. On the other hand, with proper dye and polymer chemistry, polymers that efficiently emit visible

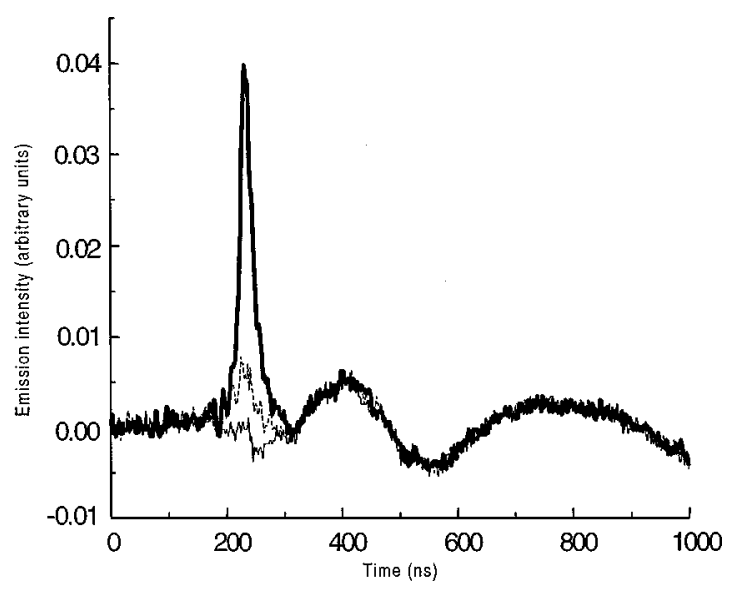

FIG. 4. Visible light emission excited in a $2 \times 10^{-4} \mathrm{M}$ solution of pyrromethene in methanol by simultaneous two-photon absorption of pump light at 1064 and $805 \mathrm{~nm}$. Bold solid line, both pump sources presented simultaneously. Note the strong visible emission. Dotted line, $805 \mathrm{~nm}$ pump only. To a small degree this wavelength is able to excite visible emission alone as evidenced by the small visible signal detected. Solid line, $1064 \mathrm{~nm}$ pump only. No visible emission is detected with this wavelength alone. light under the demonstrated excitation can be developed. Such dye-doped polymers will likely be able to serve as one-color displays. When dye/polymer combinations are more fully developed capable of blue, green, and red emission (see Table I) three-color displays will be possible. Since it is likely that unwanted intermolecular interactions will occur if all three dyes were placed in the same host, we suggest that the doped polymers could be powdered and then dispersed in a clear passive host polymer. In this model, the concentration of each emitting particulate should be such that there are many in each volume element of the display. As a result, each volume element could emit any desired color. Note that a polymer containing the dispersed particulates could be prepared in sheet form and used as a twodimensional display with single-wavelength excitation sources for each color.

The authors acknowledge support for one of the authors (E.St.M.-D.) from the National Science Foundation sponsored CREOL Summer Research Experiences for Undergraduates Program (Grant No. EEC 9732420). One of the authors (K.A.) was a summer intern at CREOL from the Ecole National Superieure de Physique de Marseille, Marseille, France. The authors thank Grant Lafontaine of CYRO Industries for providing the dye-doped plastics.

\footnotetext{
${ }^{1}$ K. Nishikawa, A. Sado, and S. Aono, Nippon Kagaku Za. 91, 829 (1970).

${ }^{2}$ W. M. McClain, Acc. Chem. Res. 7, 131 (1974).

${ }^{3}$ J. D. Lewis, C. M. Verber, and R. B. McGhee, IEEE Trans. Electron Devices ED-18, 724 (1971).

${ }^{4}$ E. Downing, L. Hesselink, J. Ralston, and R. Macfarlane, Science 273, 1185 (1996).
} 